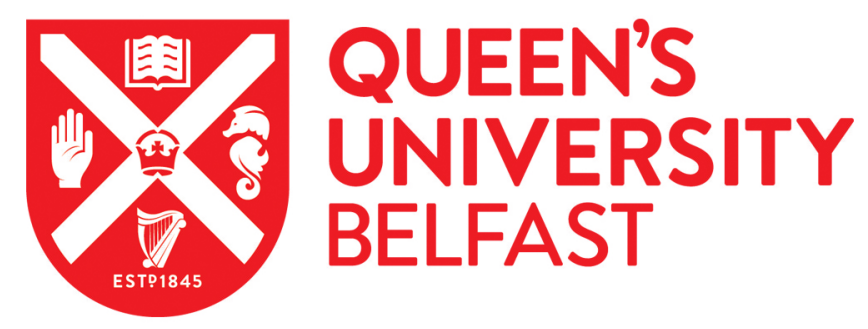

\title{
Swellable polymer films containing Au nanoparticles for point-of-care therapeutic drug monitoring using surface-enhanced Raman spectroscopy
}

Lee, W. W. Y., McCoy, C. P., Donnelly, R. F., \& Bell, S. E. J. (2017). Swellable polymer films containing Au nanoparticles for point-of-care therapeutic drug monitoring using surface-enhanced Raman spectroscopy. Analytica Chimica Acta, 912, 111-116. https://doi.org/10.1016/j.aca.2016.01.023

Published in:

Analytica Chimica Acta

Document Version:

Peer reviewed version

Queen's University Belfast - Research Portal:

Link to publication record in Queen's University Belfast Research Portal

\author{
Publisher rights \\ (C) 2016, Elsevier \\ This is an open access article published under a Creative Commons Attribution-NonCommercial-NoDerivs License \\ (https://creativecommons.org/licenses/by-nc-nd/4.0/), which permits distribution and reproduction for non-commercial purposes, provided the \\ author and source are cited.
}

\section{General rights}

Copyright for the publications made accessible via the Queen's University Belfast Research Portal is retained by the author(s) and / or other copyright owners and it is a condition of accessing these publications that users recognise and abide by the legal requirements associated with these rights.

Take down policy

The Research Portal is Queen's institutional repository that provides access to Queen's research output. Every effort has been made to ensure that content in the Research Portal does not infringe any person's rights, or applicable UK laws. If you discover content in the Research Portal that you believe breaches copyright or violates any law, please contact openaccess@qub.ac.uk. 


\title{
Swellable Polymer Films Containing Au \\ Nanoparticles for Point-of-Care Therapeutic \\ Drug Monitoring using Surface-Enhanced \\ Raman Spectroscopy
}

Wendy W. Y. Lee, ${ }^{a}$ Colin P. McCoy, ${ }^{b}$ Ryan F. Donnelly ${ }^{b}$ and Steven E. J. Bell ${ }^{a *}$

\begin{abstract}
${ }^{a}$ School of Chemistry and Chemical Engineering, David Keir Building, Queen's University Belfast, Belfast, N. Ireland, UK, BT9 5AG. E-mail: s.bell@qub.ac.uk; Fax: +44 2890 382117;

Tel: +44 2890 974470. ${ }^{\mathrm{b}}$ School of Pharmacy, Medical Biology Centre, BT9 7BL.
\end{abstract}




\section{Abstract}

Large $(10 \times 10 \mathrm{~cm})$ sheets of SERS active polymer have been prepared by stabilising metal nanoparticle aggregates within dry hydroxyethylcellulose (HEC) films. In these films the aggregates are protected by the polymer matrix during storage but in use they are released when aqueous analyte droplets cause the films to swell to their gel form. The fact that these "PolySERS" films can be prepared in bulk but then cut to size and stored in air before use means that they provide a cost effective and convenient method for routine SERS analysis. Here we have tested both $\mathrm{Ag}$ and $\mathrm{Au}$ Poly-SERS films for use in point-of-care monitoring of therapeutic drugs, using phenytoin as the test compound. Phenytoin in water could readily be detected using Ag Poly-SERS films but dissolving the compound in phosphate buffered saline (PBS) to mimic body fluid samples caused loss of the drug signal due to competition for metal surface sites from $\mathrm{Cl}^{-}$ions in the buffer solution. However, with Au Poly-SERS films there was no detectable interference from $\mathrm{Cl}^{-}$and these materials allowed phenytoin to be detected at $1.8 \mathrm{mg} \mathrm{L}^{-1}$, even in PBS. The target range of detection of phenytoin in therapeutic drug monitoring is $10-20 \mathrm{mg}$ $\mathrm{L}^{-1}$. With the Au Poly-SERS films, the absolute signal generated by a given concentration of phenytoin was lower for the films than for the parent colloid but the SERS signals were still high enough to be used for therapeutic monitoring, so the cost in sensitivity for moving from simple colloids to films is not so large that it outweighs the advantages which the films bring for practical applications, in particular their ease of use and long shelf life.

Keywords: Surface-enhanced Raman spectroscopy, metal nanoparticles, polymer, hydroxyethylcellulose, phenytoin, therapeutic drug monitoring.

\section{Introduction}

For most therapeutic drugs using a standard dose regime gives sufficient control over the level of drug in each patient. However, in some cases the toxicity of the drug and/or a narrow therapeutic window means that it is necessary to measure the drug level at regular intervals. 
Currently this is typically done through time consuming, laboratory-based analysis of blood samples with HPLC ${ }^{[1]}{ }^{[2]}$ although a numerous other methods for therapeutic drug monitoring which exploit bead technologies, enzymes, antibodies and magnetic separation in various combinations have been developed over the years. ${ }^{[3]}$

The ability to carry out therapeutic drug monitoring at the patient's bedside could potentially reduce delays associated with laboratory -based analysis and reduce costs. SERS is attractive for bedside drug monitoring since it is expected to have the required sensitivity ${ }^{[4-6]}$ and can be combined with hand-held instruments for signal readout within a matter of a few minutes. However, for such applications simple metal colloids are unsuitable as the enhancing media due to their limited shelf life and the need to have liquid sample handling steps in the analysis. Previous work ${ }^{[7-9]}$ has demonstrated that SERS could be used for the point-of-care monitoring of drugs based on tubing which could be used both to collect the sample and to detect the targets using integrated SERS enhancing components based on Ag nanodomes. This method was impressive, however the devices were complex and expensive to fabricate. Here we explore a much lower cost approach based on SERS-enhancing nanoparticle aggregates which are stabilised by encapsulation within dry swellable polymer matrices but are released on application of the analyte. Previous attempts to prepare Ag nanoparticles in polymer hosts have included the photoreduction of matrix-stabilised Ag halides ${ }^{[10]}$ and chemical reduction of agarose gels containing Ag nitrate with borohydride. ${ }^{[11]} \mathrm{Au}$ nanoparticles have also been isolated within a thiolated-polystyrene matrix. ${ }^{[12]}$ These methods each have their own disadvantages so that none have been widely adopted.

We have recently reported that large, thin $(50 \mu \mathrm{m})$ dry polymer sheets containing numerous SERS active Ag nanoparticle aggregates can be prepared by drying aqueous mixtures of hydroxyethylcelloulose (HEC) and pre-aggregated Ag colloid ${ }^{[13]}$ which was an improvement of our earlier method which used polycarbophil as dried films or gels. ${ }^{[14][15]}$ Although conventional aqueous colloids are known to give the highest SERS enhancements and are inexpensive to prepare, they are unstable systems, need to be stored at $4{ }^{\circ} \mathrm{C}$ and should not be 
stored for long periods. In the dry "Poly-SERS" films the particle aggregates are stabilised in the polymer matrix. The films can be stored at RT, are portable and are easy to handle, for example, they can be cut to size with scissors meaning they are highly appropriate for regular or intermittent bed-side SERS analysis if used alongside a portable Raman spectrometer. ${ }^{[13]}$

The Poly-SERS films are made of highly swellable HEC polymer and so upon activation with aqueous analyte, the films can rapidly absorb the analyte whilst simultaneously releasing the Ag nanoparticle aggregates to interact and generate SERS. The ability of the polymer to swell quickly means that a SERS signal can be obtained almost immediately once analyte is deposited onto the surface of the film. Furthermore, if the analyte is allowed to preconcentrate onto the film there is an increase in signal intensity with time where maximum SERS signals are given when all of the solvent evaporates. For droplets of analyte dissolved in $25 \%$ ethanol in water, optimum SERS signals can be obtained at approximately 60 minutes after depositing onto the film, i.e. when the solvent has fully evaporated.

Here we explore the use of such films for the detection of phenytoin, an anticonvulsant which is commonly used as an antiepileptic drug, in PBS as a model for interstitial fluid. Phenytoin must be routinely monitored in patients due to the adverse side effects which occur at concentrations above the target therapeutic range of 10-20 $\mathrm{mg} \mathrm{L}^{-1}$; effects include depression of the central nervous system, which can give symptoms ranging from mild sedation to coma. $[16]$

\section{Materials and methods}

\subsection{Reagents and materials}

Gold (III) chloride hydrate, magnesium sulphate, trisodium citrate, phenytoin and phosphate buffered saline (PBS) tablets were purchased from Sigma Aldrich. Natrosol hydroxyethylcellulose (HEC) 250 pharm HX was purchased from Ashland Inc. 


\subsection{Preparation of Ag Poly-SERS films}

The Ag Poly-SERS films were prepared as described previously. ${ }^{[13]}$ In brief, citrate reduced $\mathrm{Ag}$ colloids were prepared and had $\lambda_{\max }=405 \mathrm{~nm}$. The colloid was then pre-aggregated using $\mathrm{MgSO}_{4}$ mixed with HEC powder and dried to a film. The UV-vis spectrum of an $\mathrm{Au}$ Poly-SERS film showed a broad extinction from 300 to $1000 \mathrm{~nm}$ (see Supplementary Data, Fig. S1), which was due to a combination of light scattering by the semi-opaque film and absorption by the aggregated particles.

\subsection{Preparation of Au Poly-SERS films}

Colloidal Au nanoparticles ( $c a .50 \mathrm{~nm}$ diameter) were prepared by citrate reduction of gold chloride hydrate, ${ }^{[17]}$ and had $\lambda_{\max }=545 \mathrm{~nm}$. The Au Poly-SERS films where then prepared by pre-aggregating the as-prepared colloid $(54.2 \mathrm{~mL})$ with $10.8 \mathrm{~mL} \mathrm{MgSO}_{4}$ at $0.1 \mathrm{~mol} \mathrm{dm}^{-3}$ and mixing with HEC powder ( $1.2 \%$ w/v polymer) at $400 \mathrm{rpm}$ using a mechanical stirrer until a smooth, glossy gel was obtained, which typically took approx. $50 \mathrm{~min}$. The resulting gel was then poured into a $10 \times 10 \times 0.5 \mathrm{~cm}$ casting mould and left to dry in air (relative humidity $\leq 50 \%)$.

\subsection{SERS analysis with Poly-SERS films}

The Ag and Au Poly-SERS films were both used in the same way; small $(5 \times 5 \mathrm{~mm})$ squares of the films were placed onto a glass slides covered in aluminium foil before they were rehydrated by applying a $15 \mu \mathrm{L}$ aqueous droplet of analyte onto the surface of the SERS films. The aqueous analyte was then allowed to dry before a spectrum was collected by directing the probe laser from a PerkinElmer RamanMicro 200 Raman microscope using a 4× objective lens at $55 \%$ laser power onto it. The microscope uses a $785 \mathrm{~nm}$ cavity diode laser ( $80 \mathrm{~mW})$ with a Czerny-Turner spectrometer with a spectral range of $95-3200 \mathrm{~cm}^{-1}$. It is composed of an Olympus microscope (BX51 reflected illumination frame) which is connected to a spectrometer through fibre optic cables, this gives a spot diameter of $230 \mu \mathrm{m}$. In all cases, SERS spectra were collected with accumulation times of $30 \mathrm{~s}$. 
Phenytoin stock solution was initially prepared in ethanol and serial dilutions were then carried out using DDI water or PBS solution.

\subsection{SERS analysis with aqueous Au colloid}

Aqueous samples were held in 96 well polyethylene microlitre plates analysed using a $785 \mathrm{~nm}$ Avalon RamanStation. The system has a $785 \mathrm{~nm}$ laser source ( $c a .160 \mathrm{~mW}$ at sample) with a spot diameter $c a .200 \mu \mathrm{m}$. and is fitted with an echelle spectrograph. For SERS analysis, $100 \mu \mathrm{L}$ colloid was mixed with $100 \mu \mathrm{L}$ analyte solution and then aggregated with $50 \mu \mathrm{L}$ of 0.1 mol dm ${ }^{-3} \mathrm{MgSO}_{4}$ before a spectrum was recorded.

\section{Results and discussion}

Highly swellable Ag Poly-SERS films have been shown to work well with a range of organic and inorganic target molecules in previous work and their properties have been well characterised. ${ }^{[13]}$ The SEM image in Fig. 1 shows that the Ag aggregates are distributed approximately evenly across the film. 


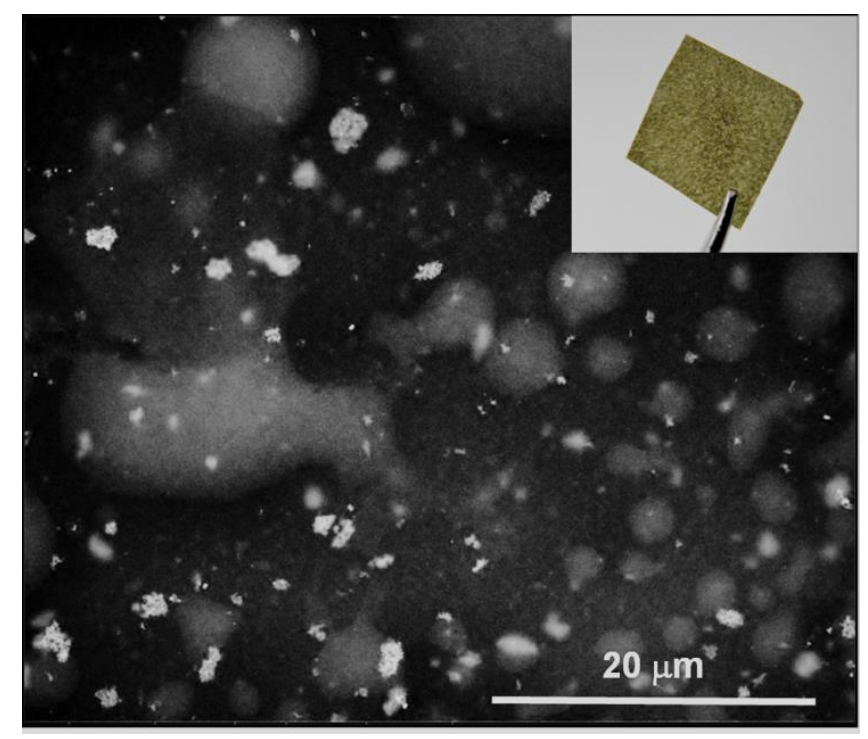

Fig. 1: SEM image showing Ag aggregates within a Poly-SERS film at 5400× magnification. The insert shows a photograph of a section of Ag Poly-SERS film cut to approximately $1 \mathrm{~cm}^{2}$.

Initial experiments on phenytoin detection were therefore carried out using these $\mathrm{Ag}$ materials. Fig. 2 (a) shows the SERS spectrum obtained when $15 \mu \mathrm{L}$ of $1 \times 10^{-4} \mathrm{~mol} \mathrm{dm}^{-3}$ phenytoin in water was deposited on an Ag Poly-SERS film. The spectrum shows characteristic bands for phenytoin which are also detected with simple aqueous Ag colloid (see Fig. 3). Although aqueous phenytoin could clearly be detected with the Ag Poly-SERS films it was important to test if this was also the case under conditions relevant to therapeutic drug monitoring. Since most body fluids including blood and interstitial fluid have high levels of salts, particularly $\mathrm{NaCl}, \mathrm{PBS}$ was used as mimic for body fluids for in vitro studies. It was found that when the phenytoin was dissolved in PBS rather than water the drug bands were no longer visible and the spectrum closely resembled that of the simple Ag colloid blank (see Supplementary Data, Fig S2). 


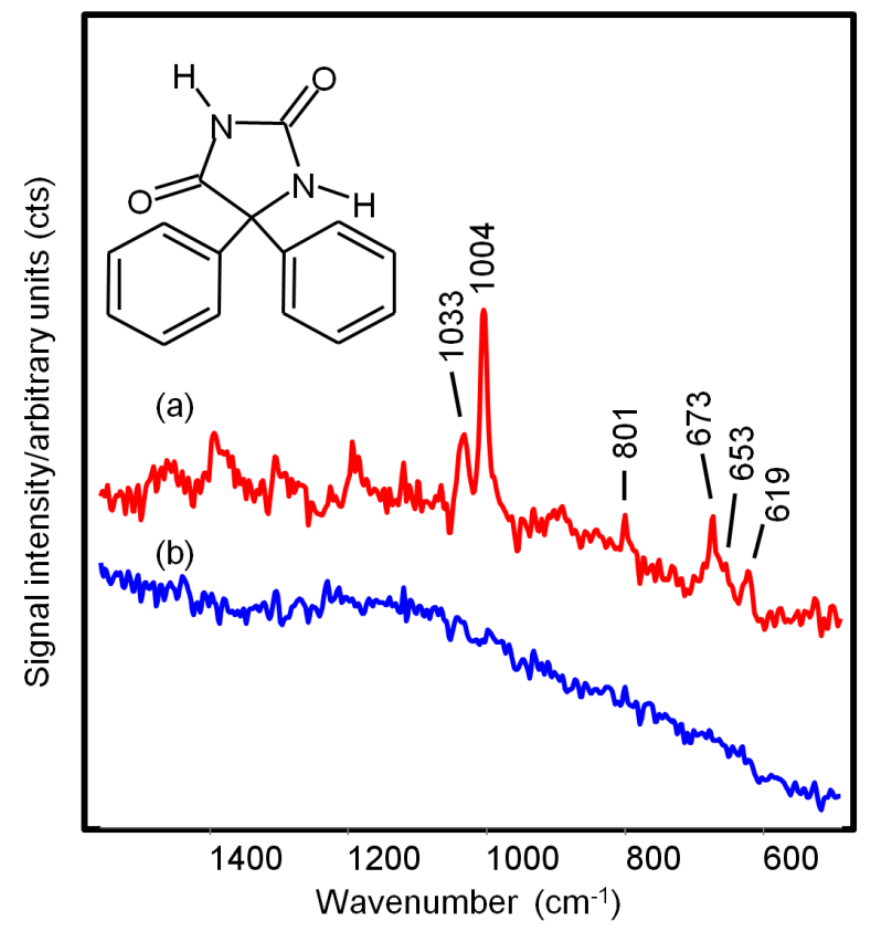

Fig. 2: SERS spectra of $15 \mu \mathrm{L}, 1 \times 10^{-4} \mathrm{~mol} \mathrm{dm}^{-3}$ phenytoin recorded using Ag Poly-SERS films. Phenytoin was dissolved in (a) water and (b) PBS. The spectra are on the same vertical scale, apart from being offset for clarity.

The loss of phenytoin bands in the presence of PBS was presumably due to competition by chloride from the buffer for $\mathrm{Ag}$ surface sites. It is known that $\mathrm{Cl}^{-}$has a high affinity for $\mathrm{Ag}$ nanoparticles ${ }^{[18-20]}$ and the low frequency region of the spectrum with PBS shows a strong Ag$\mathrm{Cl}$ band at $241 \mathrm{~cm}^{-1}$ (See Supplementary data Fig. S3). The loss of drug signal in PBS was not due to the presence of the HEC within the Poly-SERS films since the same result was seen for phenytoin and PBS with simple aqueous Ag colloid, as shown in Fig. 3. 


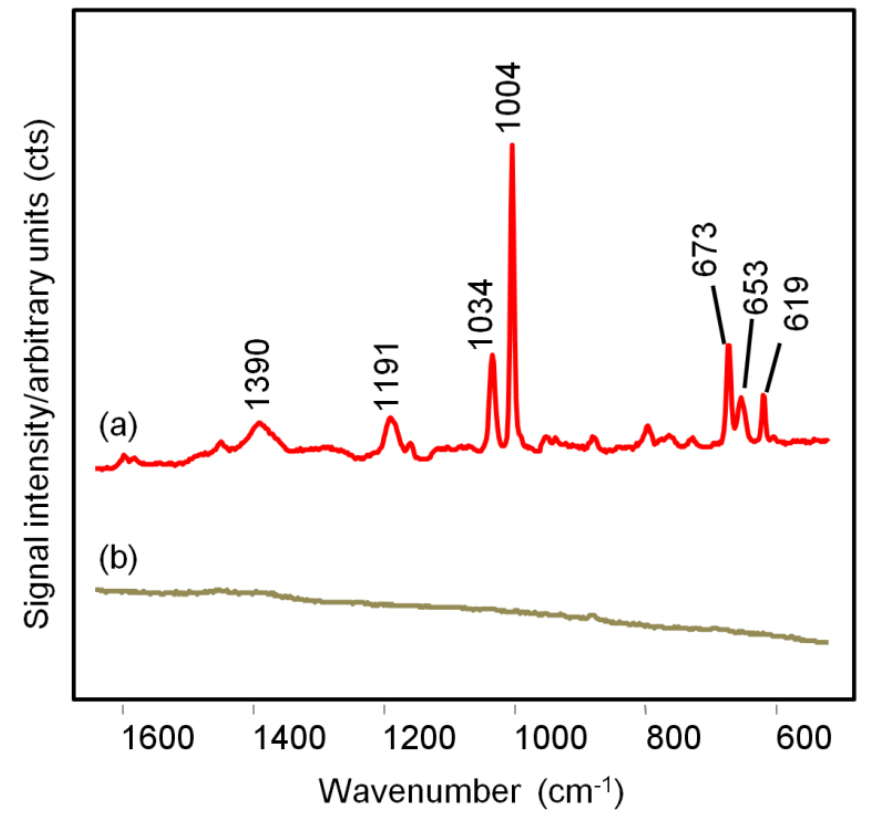

Fig. 3: SERS spectra of $1 \times 10^{-4} \mathrm{~mol} \mathrm{dm}^{-3}$ phenytoin in (a) water and (b) PBS adsorbed onto $100 \mu \mathrm{L}$ aqueous CRSC and aggregated with $0.1 \mathrm{~mol} \mathrm{dm}^{-3} \mathrm{MgSO}_{4}$. The spectra are on the same vertical scale, apart from being offset for clarity.

In an attempt to overcome this problem, aqueous colloidal Au was tested since it has a lower affinity for $\mathrm{Cl}^{-}$than colloidal $\mathrm{Ag}$ but still gives high levels of SERS enhancement. ${ }^{[17][21]}$ The spectra of phenytoin in water and in PBS obtained with $50 \mathrm{~nm}$ diameter Au colloid are shown in Fig. 4 where it is clear that there is no difference in the absolute signal height of the main phenytoin bands and therefore no $\mathrm{Cl}^{-}$interference. The enhancement factor was estimated as $3 \times 10^{4}$. An obvious approach was therefore to prepare Poly-SERS films with $\mathrm{Au}$ nanoparticles. 


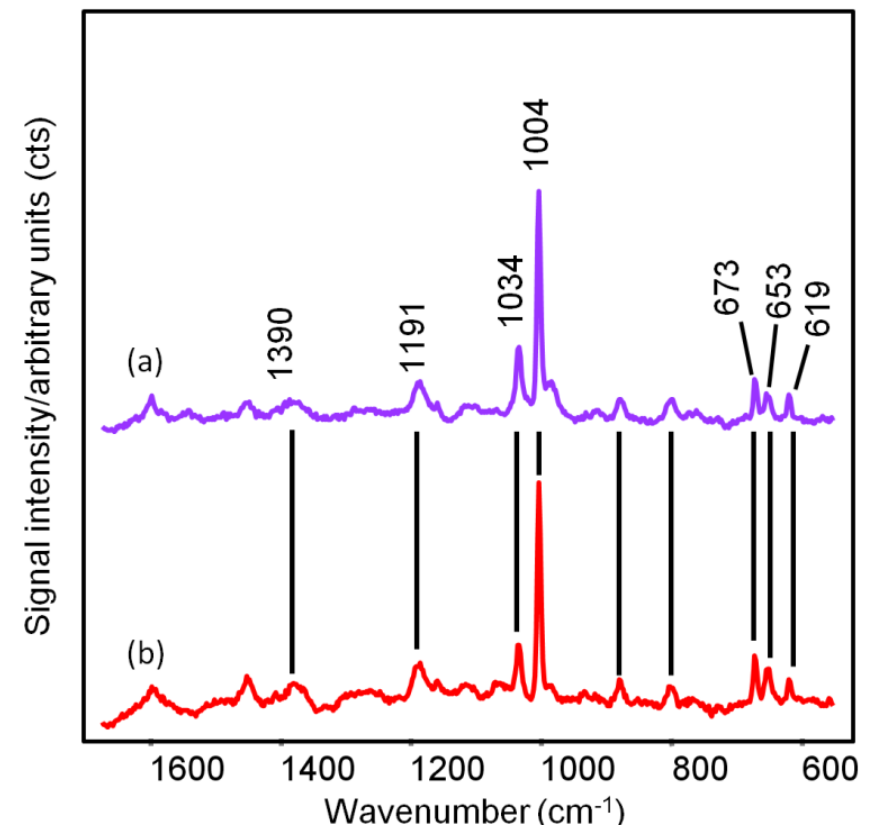

Fig. 4: SERS spectra of $1 \times 10^{-4} \mathrm{~mol} \mathrm{dm}^{-3}$ phenytoin in (a) water and (b) PBS adsorbed onto $100 \mu \mathrm{L}$ aqueous $\mathrm{Au}$ colloid and aggregated with $0.1 \mathrm{~mol} \mathrm{dm}^{-3} \mathrm{MgSO}_{4}$. Both spectra have blank backgrounds subtracted. The spectra are on the same vertical scale, apart from being offset for clarity.

It was found that the Au colloid could be isolated in HEC films in a similar way to the $\mathrm{Ag}$ colloid i.e. by pre-aggregating the colloid with $\mathrm{MgSO}_{4}$ then mixing the aggregated solution with the polymer to make a gel which was placed in a mould and allowed to dry to a flexible thin film. ${ }^{[13]}$ The resulting Au Poly-SERS films had similar physical properties to the Ag PolySERS films, ${ }^{[13]}$ in that they were flexible opaque, coloured sheets. The only difference between the materials was that the Au Poly-SERS films were purple/blue while the Ag films were a dull opaque green/brown. This purple/blue colour is characteristic of aggregated Au nanoparticles, as opposed to the red-brown colour of the starting unaggregated Au nanoparticles. The UV-vis spectrum of an Au Poly-SERS film showed a broad extinction from 300 to $1000 \mathrm{~nm}$ (see Supplementary Data, Fig. S1), as with Ag Poly-SERS films this was due to a combination of light scattering by the semi-opaque film and absorption by the aggregated particles. Figures 5 and 6 illustrate that the Au particles in the Au Poly-SERS films were in the form of dispersed 
aggregates. The average diameter of the aggregates was $c a .550 \mathrm{~nm}$ but a range of diameters was observed, the aggregates were distributed randomly in all 3 dimensions across the films. A plot showing the distribution of aggregate sizes is shown in the Supplementary Data, Fig. S4.

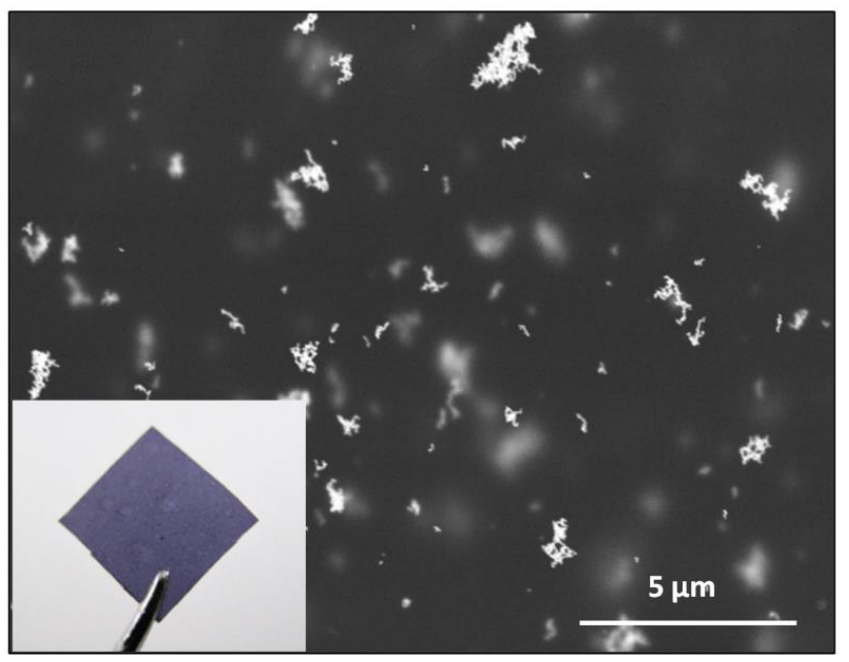

Fig. 5: SEM image showing Au aggregates within a Poly-SERS film at 14 000× magnification. The out of focus clusters are deeper within the film. The insert shows a photograph of a section of Au Poly-SERS film cut to approximately $1 \mathrm{~cm}^{2}$.

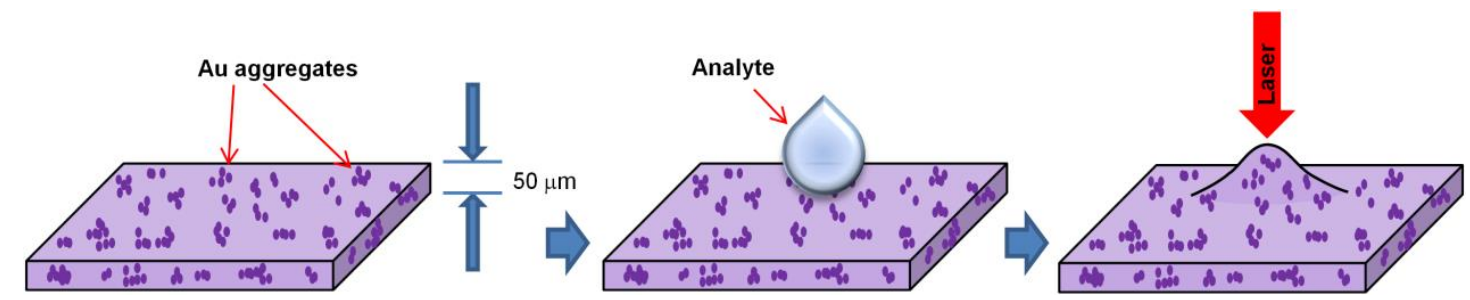

Fig. 6: Schematic diagram illustrating (a) the isolation of Au nanoparticle aggregates at various depths within a Poly-SERS film. In use, (b) an aqueous droplet of analyte is deposited onto the surface of the film, causing it to swell. (c) A SERS spectrum is then recorded using the probe laser. 
Figure 7 shows data from the Au Poly-SERS films tested with phenytoin in the absence and presence of PBS. In contrast to the previous results with Ag Poly-SERS films, there was no difference in the phenytoin signal intensities when PBS was used in place of water. The only disadvantage of the Au films was that the zero background spectrum did have stronger features than those found with the Ag films (see Supplementary Data Fig. S5). The background given with Au Poly-SERS film was very similar to that seen with the parent Au colloid, with only a small number of additional SERS features which were possibly caused by an additive/impurity within the HEC powder. Although the origin of this Au colloid background has not been identified within the SERS community, since it was not displaced by adding HEC or phenytoin, this meant that it was strongly bound to the Au surface, unlike the citrate peaks seen with aqueous Ag colloid which were easily displaced by small concentrations of chloride ions, possibly from HEC (see Supplementary data Fig. S3). Previous attempts to displace the surface species on Au colloid with excess chloride, bromide, acid, base and peroxide have all proved unsuccessful. ${ }^{[22]}$ Nevertheless, the background seen with Au PolySERS films was reproducible and did not interfere with the most dominant phenytoin band at $1004 \mathrm{~cm}^{-1}$ and in addition it could be subtracted out if required.

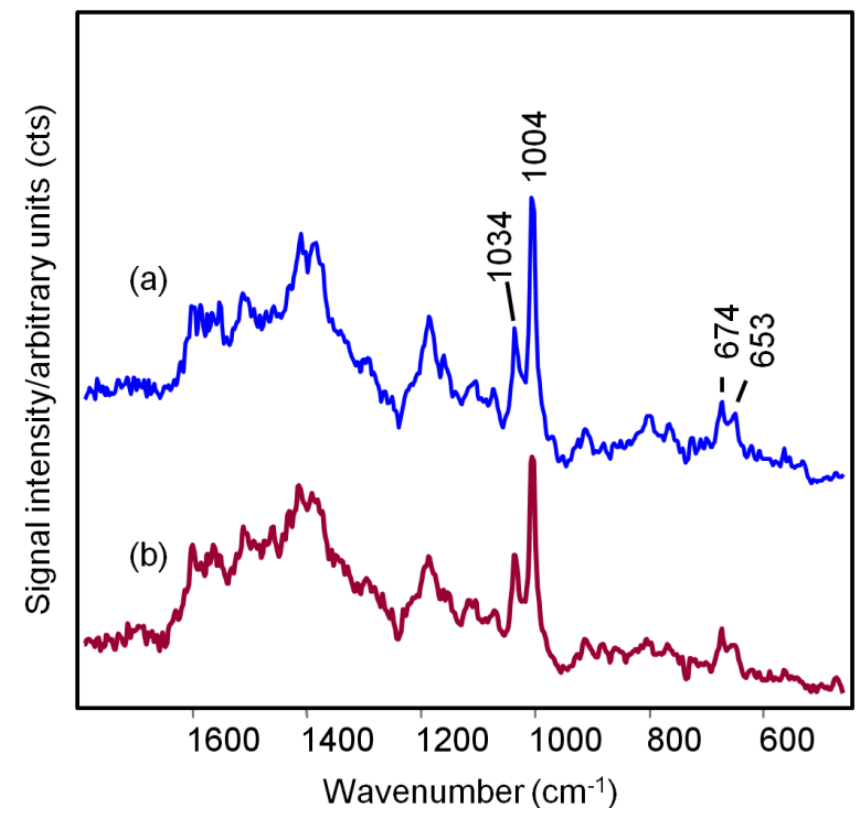


Fig. 7: SERS spectra obtained with Au Poly-SERS films of $15 \mu \mathrm{L}$ droplets of $1 \times 10^{-3} \mathrm{~mol} \mathrm{dm}^{-}$ ${ }^{3}$ phenytoin in (a) water and (b) PBS. Both spectra have blank backgrounds subtracted. The spectra are on the same vertical scale, apart from being offset for clarity.

The Au Poly-SERS films allowed phenytoin dissolved in PBS to be detected at a concentration as low as $1.8 \mathrm{mg} \mathrm{L}^{-1}\left(7 \times 10^{-6} \mathrm{~mol} \mathrm{dm}^{-3}\right)$, which is well below its target therapeutic range of 10-20 mg L $\mathrm{m}^{-1}$. Fig. 7 shows a calibration plot of phenytoin, dissolved in PBS using $\mathrm{Au}$ Poly-SERS films. The data show a strong correlation between the concentration of phenytoin and the absolute SERS signals of the most dominant $1004 \mathrm{~cm}^{-1}$ phenytoin band. As with the $\mathrm{Ag}$ Poly-SERS films, ${ }^{[13]}$ the Au Poly-SERS films were sufficiently homogenous for the current purpose, the standard deviation in the absolute SERS signal intensities measured for the same surface ( 3 spectra per measurement) with $1 \times 10^{-4} \mathrm{~mol} \mathrm{dm}^{-3}$ phenytoin was $5.5 \%$. Similarly, the films have a useful shelf life; the data shown in Fig. 8 were obtained with films which had been stored in air for $c a .9$ months after preparation.

Finally, it is interesting to compare the performance of the Poly-SERS films against their parent colloid. In the case of Ag Poly-SERS films it was found that the absolute signal heights given by the films and colloid were within a factor of 3 over a broad range of concentrations. In part, this similarity may have been the result of factors which increase or decrease the enhancement acting together in the films. For example, the polymer would be expected to hinder, at least to some extent, the access of the analyte to the enhancing surface of the particles. However, during the preparation of the Poly-SERS films they are loaded with a much higher density of particle aggregates than are present in the parent colloid which resulted in increased scattering. In other words, the dried Poly-SERS films were only approx. $50 \mu \mathrm{m}$ thick, but they contained the same number of particles as the $5 \mathrm{~mm}$ depth of gel which was initially used to prepare them. In comparison, with the aqueous colloid many of these particles will lie outside the focal depth of the system and therefore not contribute to the signal. This 
effect means that the SERS signals for strongly binding analytes which are able to overcome HEC interference give greater SERS signal intensities when using Poly-SERS films than with the aqueous colloid. ${ }^{[13]}$ Of course, phenytoin is not a typical test analyte for SERS experiments since it is not known to have strong affinities for Ag or Au colloids. Other analytes such as thiophenol interact with $\mathrm{Ag}$ or Au nanoparticles to a greater extent and so will be able to overcome interference from HEC and retain a large amount of sensitivity when tested with Poly-SERS films, in comparison to when its parent aqueous colloid is used, (see Supplementary Data, Fig. S6). In contrast, with phenytoin and Au Poly-SERS films, the absolute signal generated by a given concentration of phenytoin was found to be much lower for the films than for the parent colloid. This could be due to either the aggregates giving lower enhancement in the films or, as mentioned previously, the polymer hindering access of the analyte to the particles. These effects can be separated by considering the data for the signal intensities given by Au colloid alongside that for the films, as shown in Fig. 8. In Fig. 8 the maximum signal intensities given by both systems are similar but the phenytoin concentration which gives these signals is much lower for the aqueous colloid than the films. Indeed the aqueous colloid signals start to plateau at concentrations which are at the low end of the films' range. This implies that the particles in the films and colloid will both provide similar levels of enhancement but that higher analyte concentrations are required to reach a given signal level, presumably due to interference by the polymer matrix. In the semi-log plot, the data for the films and colloid are offset by approximately $2 \log$ units of concentration, implying that a hundredfold increase in concentration is needed to force the same amount of analyte onto the film-bound particles as onto the simple colloid. Although this appears to be a large number, since it effectively corresponds to a reduction in sensitivity of 2 orders of magnitude, the SERS signals given with Au colloid by phenytoin are so high that even with this reduction in the sensitivity the signals given by the films remain high enough to be used for therapeutic drug monitoring, as shown by the line marked on the Figure. This means that the cost in sensitivity for moving from simple colloids to films is not so large that it outweighs the advantages which the films bring, in particular their ease of use and long shelf life. 


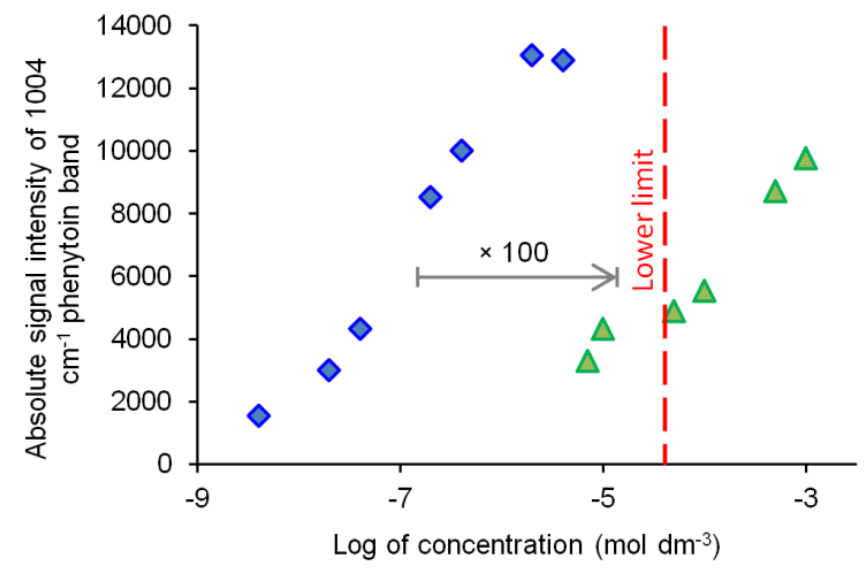

Fig. 8: Plot comparing the absolute SERS signal intensities of phenytoin in PBS obtained with aggregated aqueous Au colloid (blue) and Au Poly-SERS films which had been stored in air for $c a .9$ months (green). The lower limit of the target therapeutic range for phenytoin has been highlighted as a dashed line.

\section{Conclusions}

Interference by $\mathrm{Cl}^{-}$ions prevents the use of $\mathrm{Ag}$ colloids or films prepared from $\mathrm{Ag}$ colloids for monitoring phenytoin. Au colloids are less susceptible to interference by $\mathrm{Cl}^{-}$and here it was found that HEC films could be used to stabilise Au aggregates to give Au PolySERS substrates. These materials have the same advantages of long shelf life and ease of use as the previously reported $\mathrm{Ag}$ analogues but without the same problems with $\mathrm{Cl}^{-}$interference for weakly adsorbing analytes such as phenytoin. Encapsulation of the Au reduces the sensitivity of the Au Poly-SERS films compared to their parent colloid but the sensitivity is still sufficient for therapeutic monitoring of phenytoin.

\section{Acknowledgements}

Support for this work by the EPRSC (grant number EP/H021647/1) is acknowledged. 


\section{References}

[1] Z. Rezaei, B. Hemmateenejad, S. Khabnadideh, M. Gorgin, Simultaneous spectrophotometric determination of carbamazepine and phenytoin in serum by HPLC regression and comparison with HPLC, Talanta 65 (2005) 21-28.

[2] N. A. Santagati, R. Gotti, G. Rosisvalle, Simultaneous determination of phenytoin and dextromethophan in urine by solid-phase extraction and HPLC-DAD, J. Sep. Sci. 28 (2005) $1157-1162$.

[3] http://www.healthcare.siemens.co.uk/siemens_hwem-hwem_ssxa_websites-contextroot/wcm/idc/groups/public/@global/@clinicalspec/documents/download/mdaw/mtu3/ edisp /tdm_guide_final-00028657.pdf (accessed August 17, 2014)

[4] S. Nie, S. R. Emory, Probing single molecules and single nanoparticles by surface-enhanced Raman scattering, Science 275 (1997) 1102-1106.

[5] S. E. J. Bell, N. M. S. Sirimuthu, Quantitative surfaced-enhanced Raman spectroscopy, Chem. Soc. Rev. 37 (2008) 1012-1024

[6] S. E. J. Bell, Quantitative analysis of solid dosage formulations by Raman spectroscopy, Pharmaceutical applications of Raman spectroscopy, in: S. Sasic (Eds.), John Wiley \& Sons, New Jersey, 2008, pp. 29-60.

[7] C. J. Choi, H. Wu, S. George, J. Weyhenmeyer, B. T. Cunningham, Biochemical sensor tubing for point-of-care monitoring of intravenous drugs and metabolites, Lab Chip 12 (2012) $574-581$.

[8] C. J. Choi, H. Wu, B. T. Cunningham, SERS sensors for biomedical tubing, Procedia Eng. 25 (2011) 76-79. 
[9] H. Wu, B. T Cunningham, Point-of-care detection and real-time monitoring of intravenously delivered drugs via tubing with an integrated SERS sensor, Nanoscale 6 (2014) 5162-5171.

[10] B. Sagmuller, B. Schwarze, G. Brehm, S. Schneider, Application of SERS spectroscopy to the identification of (3,4-methylenedioxy) amphetamine in forensic samples utilizing matrix stabilized silver halides, Analyst 126 (2001) 2066-2071.

[11] M. Keating, Y. Chen, I. A. Larmour, K. Faulds, D. Graham, Growth and surface-enhanced Raman scattering of Ag nanoparticle assembly in agarose gel, Meas. Sci. Technol. 23 (2012) $1-9$.

[12] M. K. Corbierre, N. S Cameron, M. Sutton, S. G. J. Mochrie, L. B. Lurio, A. Rühm, R. B. Lennox, Polymer-stabilized gold nanoparticles and their incorporation into polymer matrices, J. Am. Chem. Soc. 123 (2001) 10411-10412.

[13] W. W. Y. Lee, V. A. D. Silverson, C. P.; McCoy, R. F. Donnelly, S. E. J. Bell, Preaggregated Ag nanoparticles in dry swellable gel films for off-the-shelf surface-enhanced Raman spectroscopy, Anal. Chem. 86 (2014) 8106-8113.

[14] S. E. J. Bell, S. J. Spence, Disposable, stable media for reproducible surface-enhanced Raman spectroscopy, Analyst 126 (2001) 1-3.

[15] S. E. J. Bell, N.M. S. Sirimuthu, Rapid, quantitative analysis of ppm/ppb nicotine using surface-enhanced Raman scattering from polymer-encapsulated Ag nanoparticles (gel-colls), Analyst 129 (2004) 1032-1036.

[16] D. S. Aschenbrenner, S. J. Venable, Drug therapy in nursing, third ed., Lippincott Williams \& Wilkins, China, 2009, 335-332.

[17] S. E. J. Bell, M. R. McCourt, SERS enhancement by aggregated Au colloids: effect of particle size, J. Phys. Chem. 11 (2009) 7455-7462. 
[18] S. E. J. Bell, N. M. S. Sirimuthu, Surface-enhanced Raman spectroscopy as a probe of competitive binding by anions to citrate-reduced silver colloids, J. Phys. Chem. 109 (2005) 7405-7410.

[19] E. Papadopoulou, S. E. J. Bell, Structure of adenine on metal nanoparticles: pH equilibria and formation of $\mathrm{Ag}^{+}$complexes detected by surface-enhanced Raman spectroscopy, J. Phys. Chem. 114 (2010) 22644-22651.

[20] S. E. J. Bell, N. M. S. Sirimuthu, Surface-enhanced Raman spectroscopy (SERS) for submicromolar detection of DNA/RNA mononucleotides, J. Am. Chem. Soc. 128 (2006) 1558015581.

[21] S. Nath, S. K. Ghosh, S. Kundu, S. Praharaj, S. Panigrahi, T. Pal, Is gold really softer than silver? HSAB principle revisited, J. Nanoparticle Research, 8 (2006) 111-116

[22] S. Zheng, M.Phil. Thesis, Queen's University Belfast, 2012. 